\title{
Anomalies in Hyperfine Structure
}

\author{
S. Goudsmit And R. F. BACher, ${ }^{*}$ University of Michigan \\ (Received April 13, 1933)
}

\begin{abstract}
The deviations from the interval rule in the hyperfine structure of two of the energy states in mercury can be explained satisfactorily as due to perturbations arising from their proximity. The forbidden line $\mathrm{Hg}$ I $\lambda 2967.5$ $\left(6 s 6 d^{1} D_{2}-6 s 7 p^{3} P_{0}\right)$ and its hyperfine structure are also
\end{abstract}

explained by these perturbations. The hyperfine structure ascribed to the $3 s$ ff levels of ionized aluminum which has seemed to be in contradiction with the theory, can be shown to belong partially to the $3 s$ ng levels and to come out in agreement with theoretical expectations.
$T^{H}$ HE difficulties encountered in the interpretation of hyperfine structure can be divided into two classes. The first class contains those which are due to our lack of knowledge of nuclear structure. The main questions are why certain nuclei possess the observed mechanical and magnetic moments and why they cause the observed amount of isotope shift. The second class of difficulties is connected with the interaction of the nucleus with the surrounding electrons. Assuming that the hyperfine structure is due to nuclear spin, the observed splitting of the levels does not always agree with this hypothesis. For example, the values of the nuclear magnetic moments calculated from different levels of the same atom were not always the same. Similar discrepancies seemed to exist for isotope shifts. It has been shown by various workers ${ }^{1}$ that these difficulties do not mean that the hypothesis of nuclear spin is incorrect, but that they arise from neglect of important relativity corrections or from the approximate nature of certain numerical calculations.

In two more or less isolated cases no satisfactory explanation of discrepancies has been given up to the present. They are a deviation from the interval rule for the hyperfine structure of a level in neutral mercury $(\mathrm{Hg} \mathrm{I})$ and a discrepancy in the magnitude and number of components of the hyperfine structure of a few levels in ionized aluminum (Al II). It is hoped that this paper will clarify these difficulties.

* Alfred Lloyd Fellow.

${ }^{1}$ Breit, Phys. Rev. 42, 348 (1932). Racah, Zeits. f. Physik 71, 431 (1931). Goudsmit, Phys. Rev. 43, 636 (1933).

\section{MERCURY}

In Fig. 2 the crosses represent the $6 s 6 d,{ }^{3} D_{1}$ and ${ }^{1} D_{2}$ levels of $\mathrm{Hg}$ I with their hyperfine structure as given in the interpretation by Schüler and his coworkers. ${ }^{2}$ The isotopes of even atomic weight show no splitting up and also no observable isotope shift. The latter means that the isotope shift for initial and final levels of the investigated lines is practically the same, in agreement with expectations. Isotope 199 has a nuclear moment $\frac{1}{2}$ and a negative $g$-value, isotope 201 has a moment $1 \frac{1}{2}$ and a positive $g$-value. From other levels the ratio of the $g$-values is found to be $g(199) / g(201)=-2.70$. Using this ratio and the interval rule one may find that the total hyperfine structure separation of a level of $\mathrm{Hg}^{199}$ with $J=1$ is 1.01 times larger than that of $\mathrm{Hg}^{201}$. For levels with $J=2$ the total separation of $\mathrm{Hg}^{199}$ is 0.90 times that of $\mathrm{Hg}^{201}$.

Schüler and Jones point out that the interval rule is violated in $\mathrm{Hg}^{201}$, the separation of the lowest two levels being too small. They notice moreover that the center of gravity of the hyperfine splitting of the odd isotopes does not coincide with the position of the levels for the even isotopes and that the ratio of the total separations is also in disagreement with expectations. Schüler and Jones ascribe these deviations to a mutual perturbation between these two neighboring levels, which causes an apparent repulsion between levels with the same

\footnotetext{
2 See especially Schüler and Jones, Zeits. f. Physik 77, 801 (1932).
} 
quantum number $F$. We shall show that this supposition is for the most part correct. ${ }^{3}$

The perturbation in this case is similar to a beginning Paschen-Back effect since the hyper.fine separation is of the same order as the distance between the two levels. It can be treated quantitatively in the same manner as the transition from one scheme of vector coupling to another. It is possible to write down simple expressions for the energy levels in the two extreme cases where the hyperfine structure is much smaller and where it is much larger than the distance between the two levels. This knowledge is sufficient to obtain the level pattern for all intermediate cases. We shall assume that the nuclear moment interacts with the $6 s$ electron only and shall neglect its interaction with the $6 d$ electron which is estimated to be more than a hundred times smaller. We shall also neglect perturbations due to levels farther away as it is too complicated to take them into account. Such perturbations are expected to be small but may perhaps explain why we do not find exact agreement between observed and calculated levels.

The distance between the two levels in case there is no hyperfine structure is denoted by $X$ and is found from the even isotopes to be approximately $3.0 \mathrm{~cm}^{-1}$. The exact value is not necessary for our purpose. The interaction of the nuclear moment with the $6 s$ electron is of the form (1).

$$
E=A I s \cos (I, s)
$$

$I$ and $s$ are the nuclear spin and the spin of the $s$ electron, respectively. From the hyperfine structure of other states in $\mathrm{Hg} \mathrm{I}$ it is found that $A$ is approximately $1.20 \mathrm{~cm}^{-1}$ for $\mathrm{Hg}^{199}$ and -0.445 for $\mathrm{Hg}^{201}$.

${ }^{3}$ The perturbation in this case is not quite similar to that brought forward by Langer (Phys. Rev. 35, 649 (1930) and applied by Russell and Shenstone (Phys. Rev. 39, 415 (1932)) as was stated by Schüler and Jones. In that case the perturbations arose from the large nondiagonal matrix elements in the electrostatic energy matrix. Here, rather, it is a change of coupling due to the close proximity of levels with different $J$ values. This allows one to consider only $F$ the total angular momentum as a constant of the motion, and no longer $J$. That it also causes a repulsion between levels of the same total quantum number is not particularly characteristic, as this is common to all quantum mechanical perturbations.
In order to use the sum relations two extreme cases will be considered, first with the ordinary multiplet structure large compared to the hyperfine structure $(X \gg A)$ and second with the hyperfine structure large compared to the ordinary multiplet structure $(X \ll A)$. In the latter case we shall pass to the limit and take $X=0$ which means $(j, j)$ coupling. By using the strict validity of the interval rule for $X \gg A$ and the sum rule in passing from one extreme to the other, it is possible to find the energy values for the extreme cases. These energies, referred in each case to the largest $F$-value as zero, are given in Table $I$ and plotted in Fig. 1. The two isotopes must, of course, be treated separately. In comparing Fig. 1 and Table I it must be noted that for $\mathrm{Hg}^{201}, A$ is negative.

TABLE I. Energies of ${ }^{3} D_{1}$ and ${ }^{1} D_{2}$ in extreme coupling schemes.

\begin{tabular}{|c|c|c|}
\hline $\mathrm{Hg}^{199} I=\frac{1}{2}$ & $A \ll X$ & $X=0$ \\
\hline$F=2 \frac{1}{2}$ & $E=0$ & reference level \\
\hline$F=1 \frac{1}{2}$ & $\begin{aligned} E^{\prime} & =-\frac{5}{8} A \\
E^{\prime \prime} & =X-\frac{3}{8} A\end{aligned}$ & $\begin{aligned} E^{\prime} & =0 \\
E^{\prime \prime} & =-A\end{aligned}$ \\
\hline$F=\frac{1}{2}$ & $E=X$ & $E=0$ \\
\hline $\mathrm{Hg}^{201} \quad I=1 \frac{1}{2}$ & $A \ll X$ & $X=0$ \\
\hline$F=3 \frac{1}{2}$ & $E=0$ & reference level \\
\hline$F=2 \frac{1}{2}$ & $\begin{aligned} E^{\prime} & =-\frac{7}{8} A \\
E^{\prime \prime} & =X-\frac{9}{8} A\end{aligned}$ & $\begin{aligned} E^{\prime} & =0 \\
E^{\prime \prime} & =-2 A\end{aligned}$ \\
\hline$F=1 \frac{1}{2}$ & $\begin{aligned} E^{\prime} & =-3_{2}^{3} A \\
E^{\prime \prime} & =X-\frac{1}{2} A\end{aligned}$ & $\begin{aligned} E^{\prime} & =0 \\
E^{\prime \prime} & =-2 A\end{aligned}$ \\
\hline$F=\frac{1}{2}$ & $\begin{aligned} E^{\prime} & =-\frac{15}{8} A \\
E^{\prime \prime} & =X-\frac{1}{8} A\end{aligned}$ & $\begin{aligned} E^{\prime} & =0 \\
E^{\prime \prime} & =-2 A\end{aligned}$ \\
\hline
\end{tabular}

TABLE II. Calculated and observed levels of ${ }^{3} D_{1}$ and ${ }^{1} D_{2}$.

\begin{tabular}{|c|c|c|c|c|c|c|}
\hline & $F$ & calc. ${ }^{\mathrm{Hg}^{19}}$ & obs. & $F$ & calc. ${ }^{H g^{201}}$ & obs. \\
\hline \multirow{3}{*}{${ }^{3} D_{1}$} & $\frac{1}{2}$ & $0.300 \mathrm{~cm}^{-1}$ & $0.329 \mathrm{~cm}^{-1}$ & $2 \frac{1}{2}$ & $0.226 \mathrm{~cm}^{-1}$ & $0.260 \mathrm{~cm}^{-1}$ \\
\hline & $1 \frac{1}{2}$ & -0.051 & -0.056 & $1 \frac{1}{2}$ & -0.055 & -0.065 \\
\hline & & & & $\frac{1}{2}$ & -0.258 & -0.260 \\
\hline \multirow{4}{*}{${ }^{1} D_{2}$} & $2 \frac{1}{2}$ & 0.300 & 0.301 & $\frac{1}{2}$ & 0.480 & 0.460 \\
\hline & $1 \frac{1}{2}$ & -0.549 & -0.559 & $1 \frac{1}{2}$ & 0.277 & 0.279 \\
\hline & & & & $2 \frac{1}{2}$ & -0.007 & -0.022 \\
\hline & & & & $3 \frac{1}{2}$ & -0.337 & -0.335 \\
\hline
\end{tabular}

The information given in Table $I$ is sufficient to write down quadratic equations, ${ }^{4}$ the roots of which will give the positions of the levels for any intermediate value of $A$ and $X$. These equations are given in (2) and (3).

\footnotetext{
${ }^{4}$ Goudsmit, Phys. Rev. 35, 1325 (1930).
} 


$$
\begin{array}{ll}
\mathrm{Hg}^{199} & I=\frac{1}{2} \\
F=2 \frac{1}{2} & E=0 \\
F=1 \frac{1}{2} & E^{2}-(X-A) E-\frac{5}{8} X A=0 \\
F=\frac{1}{2} & E=X
\end{array}
$$

Center of gravity $G=\frac{3}{8} X+\frac{3}{4} A$

$$
\begin{array}{ll}
\mathrm{Hg}^{201} & I=1 \frac{1}{2} \\
F=3 \frac{1}{2} & E=0 \\
F=2 \frac{1}{2} & E^{2}-(X-2 A) E-\frac{7}{8} X A=0 \\
F=1 \frac{1}{2} & E^{2}-(X-2 A) E-\frac{3}{2} X A=0 \\
F=\frac{1}{2} & E^{2}-(X-2 A) E-\frac{15}{8} X A=0
\end{array}
$$

Center of gravity $G=\frac{3}{8} X+\frac{1}{4} A$.

The level schemes in Fig. 2 give the positions of the levels calculated from (2) and (3), taking $X=3.0 \mathrm{~cm}^{-1} ; A(199)=1.20 \mathrm{~cm}^{-1}$ and thus from the known $g$ ratio $A(201)=-0.445 \mathrm{~cm}^{-1}$. The crosses indicate the observed positions of the levels. Not only is there good agreement within each isotope, but the relative position of the levels of the two odd isotopes and their relation to those of the even isotopes fits very well. The outside level scheme of the figure indicates the

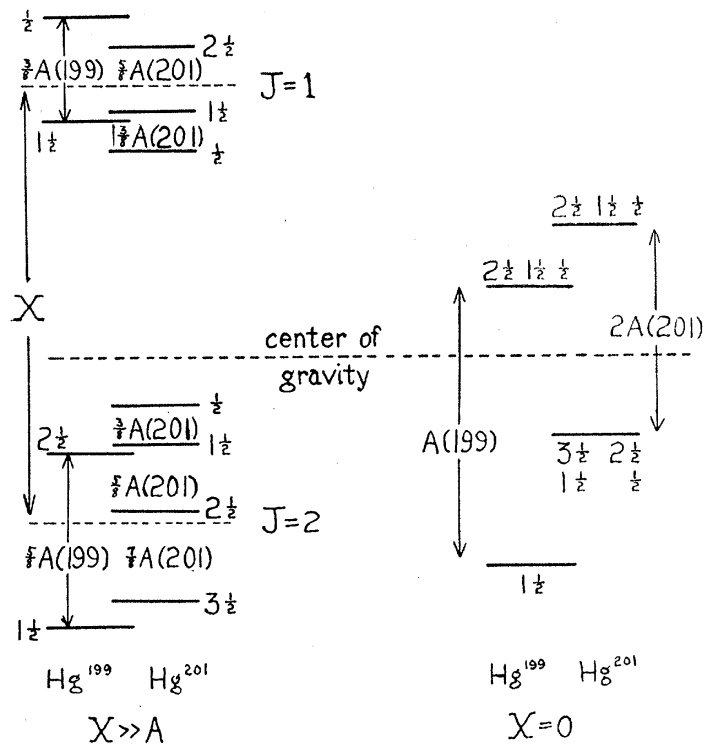

FIg. 1. Positions of $6 s 6 d{ }^{3} D_{1}$ and ${ }^{1} D_{2}$ levels of $\mathrm{Hg}$ I for $X \gg A$ and for $X=0$. For convenience the distance $X$ at the left is not drawn to scale. It should be much larger than it is drawn in the diagram. hypothetical position of the levels calculated from Table I if there were no perturbation. The small discrepancies which remain may well be due to perturbations by other levels or perhaps may be attributed to some uncertainty in the measurements of the highly complicated line patterns. Table II gives the calculated and observed levels referred to the position of the even isotopes. ${ }^{5}$

Perturbations of the type discussed here are always accompanied by anomalies in intensities and the occurrence of "forbidden" transitions. In cases where there are only two levels of the same total quantum number $F$ which perturb each other, the intensity change is given by a simple expression. Let $\delta$ denote the displacement of the level from the ideal position which it would have had without the perturbations and let $\Delta$ be the distance of this level from the other one with the same quantum number $F$, also in the ideal case. Then the ratio of the intensity of the forbidden transition $I_{1}$ to that of the allowed one $I_{0}$ is given by (4)

$$
I_{1} / I_{0}=\delta /(\Delta+\delta) .
$$

This can be derived by considering the secular determinant which gives rise to quadratic equations like those given above. One must expect therefore that for the odd mercury isotopes, transitions can occur from $6 s 6 d{ }^{1} D_{2}$ to odd levels with $J=0$ and from ${ }^{3} D_{1}$ to odd levels with $J=3$. An example is the transition ${ }^{1} D_{2}-6 s 7 p^{3} P_{0}$, $\lambda 2967.5$. Fig. 3 represents the expected transitions and indicates the intensities as obtained from (4). One sees that one can expect two or perhaps three components to have been observed. Cardaun $^{6}$ indeed gives two components 0.76

\footnotetext{
${ }^{5}$ Professor Breit has called our attention to the fact that our treatment of these mercury lines is really mathematically identical with the calculations of Casimir (Zeits. f. Physik 77, 811 (1932)). We believe, however, that our method is considerably simpler. Casimir concludes that there is grave disagreement between theory and experiment because he considers only the ratio of the displacements from the ideal positions. This ratio is too sensitive to small errors to justify Casimir's conclusion. He does not calculate the theoretical positions of the levels. Casimir's calculations are connected with ours by observing that his quantity $D=\frac{1}{4} A$. Using the values of $A$ given above, Casimir's formulas give the same final results as ours.

${ }^{6}$ Cardaun, see Kayser and Konen, Vol. VII, page 674.
} 

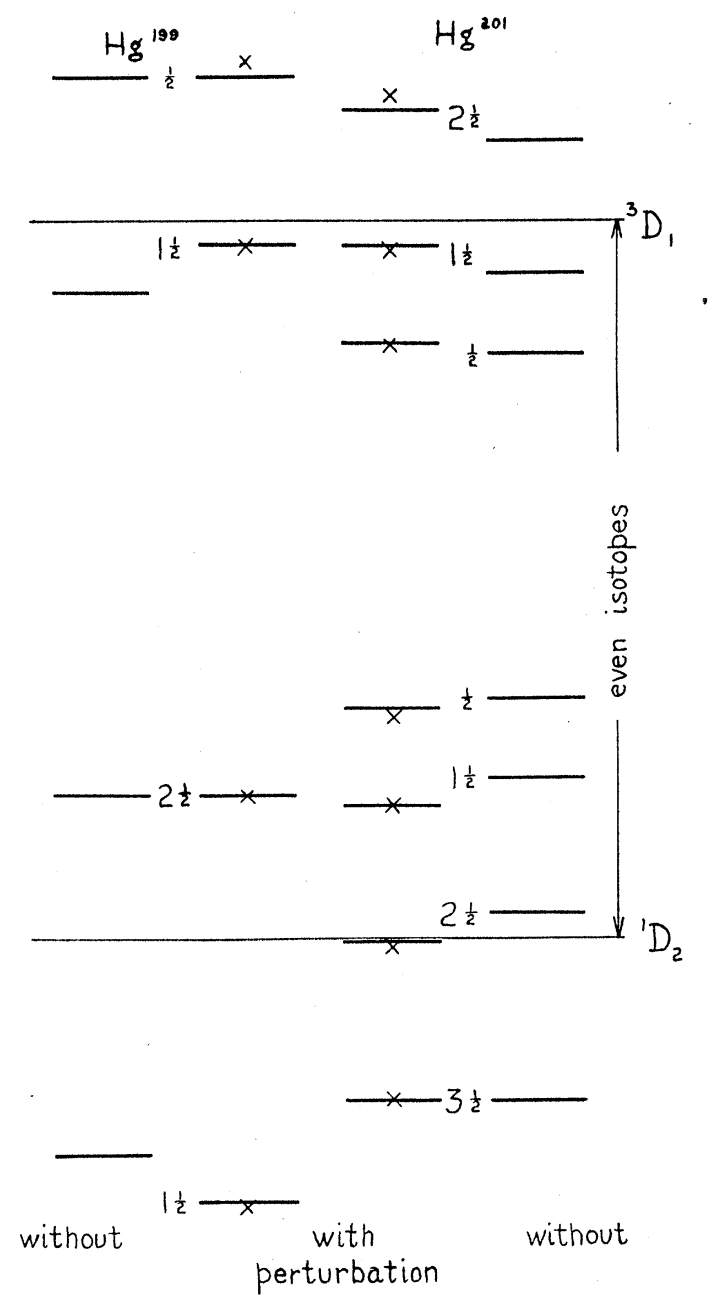

FIG. 2. Level diagram of $6 s 6 d^{3} D_{1}$ and ${ }^{1} D_{2}$ of $\mathrm{Hg} \mathrm{I}$ calculated with and without perturbation. The crosses indicate the observed levels. The distance ${ }^{3} D_{1}-{ }^{1} D_{2}$ is drawn about half its actual size, for convenience.

$\mathrm{cm}^{-1}$. apart. Wendt ${ }^{7}$ also has observed two components $(\Delta \nu=0.70)$, but his reproduction shows clearly that the one with larger frequency is broadened.

\section{Aluminum}

In his work on the classification of the spectrum of ionized aluminum Paschen ${ }^{8}$ found that the $3 s$ f ${ }^{3} F_{4},{ }^{3} F_{3},{ }^{3} F_{2}$ and ${ }^{1} F_{3}$ levels show hyperfine structure in combination with higher members of the $3 s \mathrm{n} g$ series. Since the separations were all

\footnotetext{
7 Wendt, Ann. d. Physik 37, 545 (1912).

${ }^{8}$ Paschen, Ann. d. Physik 71, 537 (1923). See also Sawyer and Paschen, Ann. d. Physik 84, 1 (1927).
}

nearly the same the structure was attributed to the hyperfine structure of the $3 s 4 f$ levels alone. This hyperfine structure was at first entirely impossible to understand as no other levels in Al II were known to show any separation. Recently, however, Ritsch ${ }^{9}$ has discovered the presence of hyperfine structure in other levels of $\mathrm{Al}$ II and Al I and has determined the nuclear spin to be $\frac{1}{2}$. From the hyperfine structure found by Ritschl one can conclude that the $3 s$ ff levels must have a hyperfine structure of the order of magnitude of that assigned by Paschen. Nevertheless this structure is in disagreement with the theory and fails to explain why the $3 s 4 f$ terms do not show the same hyperfine structure in other combinations. In Al II the hyperfine structure is caused almost entirely by the deeply penetrating $3 s$ electron present in most of the electron configurations. In such a case one expects Eq. (5) to hold. ${ }^{10}$

$$
A=\frac{1}{2} a(g-1) \text {. }
$$

$A$ is the separation factor for a given level and $a$ that of the $s$ electron. This expression is strictly valid only for Russell-Saunders coupling, $g$ being the Landé $g$-factor. The total width of the

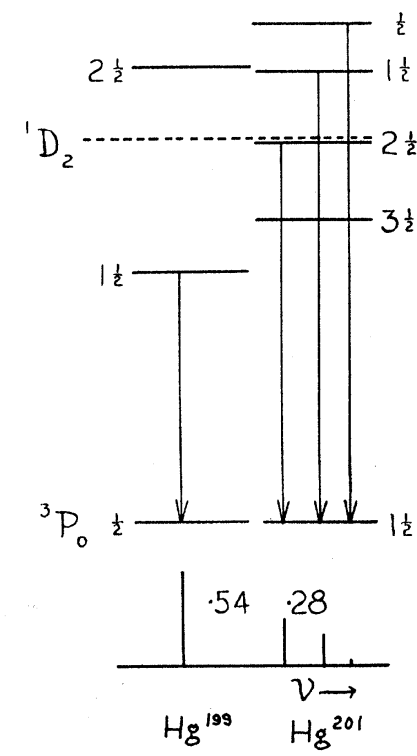

FIG. 3. Calculated structure of the forbidden line $\lambda 2967.5$ $\left(6 s 6 d^{1} D_{2}-6 s 7 p^{3} P_{0}\right)$ of $\mathrm{Hg} \mathrm{I}$.

${ }^{9}$ Ritschl, Nature 131, 58 (1933).

${ }^{10}$ Goudsmit and Bacher, Phys. Rev. 34, 1501 (1929). 
splitting of the $3 s$ ff levels should be therefore ${ }^{3} F_{4}: 9 a / 20,{ }^{3} F_{3}: 7 a / 48,{ }^{3} F_{2}:-5 a / 12,{ }^{1} F_{3}: 0$.

The observed hyperfine structures are $0.10,0.49$, $0.25,0.49 \mathrm{~cm}^{-1}$. The value for $a$ derived from Ritschl's data is about $a=0.50 \mathrm{~cm}^{-1}$ and one, would expect therefore

${ }^{3} F_{4}: 0.23,{ }^{3} F_{3}: 0.07,{ }^{3} F_{2}:-0.21,{ }^{1} F_{3}: 0 \mathrm{~cm}^{-1}$.

Though the levels of the $3 s \mathrm{n} f$ series in aluminum are greatly perturbed by other levels, these perturbations cannot be the reason for the irregularities in the hyperfine structure of the $3 s \quad 4 f$ state. The perturbing states do not contain a $3 s$ electron and possess themselves no appreciable hyperfine structure.

The discrepancy can be removed entirely if one considers first that the $3 s \mathrm{ng}$ states also must possess a hyperfine splitting due to the $3 s$ electron. The data indicate the interesting fact that for these states the hyperfine structure is larger than the multiplet splitting of the ${ }^{3} G$ as well as the singlet triplet distance from ${ }^{3} G$ to ${ }^{1} G$. We have here an extreme coupling in which the nuclear spin is more strongly coupled to the $3 \mathrm{~s}$ electron than the latter is to the $g$-electron. The hyperfine structure in this case will be that of the $3 s$ electron, as if the $g$-electron were absent, and will thus be equal to $a$. The upper part of Fig. 4 gives the level scheme for this case. It will be the same for all higher members of the $3 \mathrm{~s} n \mathrm{~g}$ series.

Special attention must be given to the selection and intensity rules for the transition from this extreme coupling to the other extreme of the $F$-states. Though it is possible to set up the intensity formulas, we believe that it is not necessary for this case to go into such complicated derivations. We shall restrict ourselves to simple correspondence considerations to find out which transitions will be strongest. In both extreme couplings the interaction between the orbit of the electron which makes the transition and the nuclear spin as well as that between the orbit and $s$-electron will be negligible compared to the other interactions. Therefore only such transitions may occur in which the position of the spin of the $s$-electron with respect to the nuclear spin is not altered. In the upper level of the $G$-state these spins are parallel, in the lower they are opposite. For the upper hyperfine levels of ${ }^{3} F_{4}$

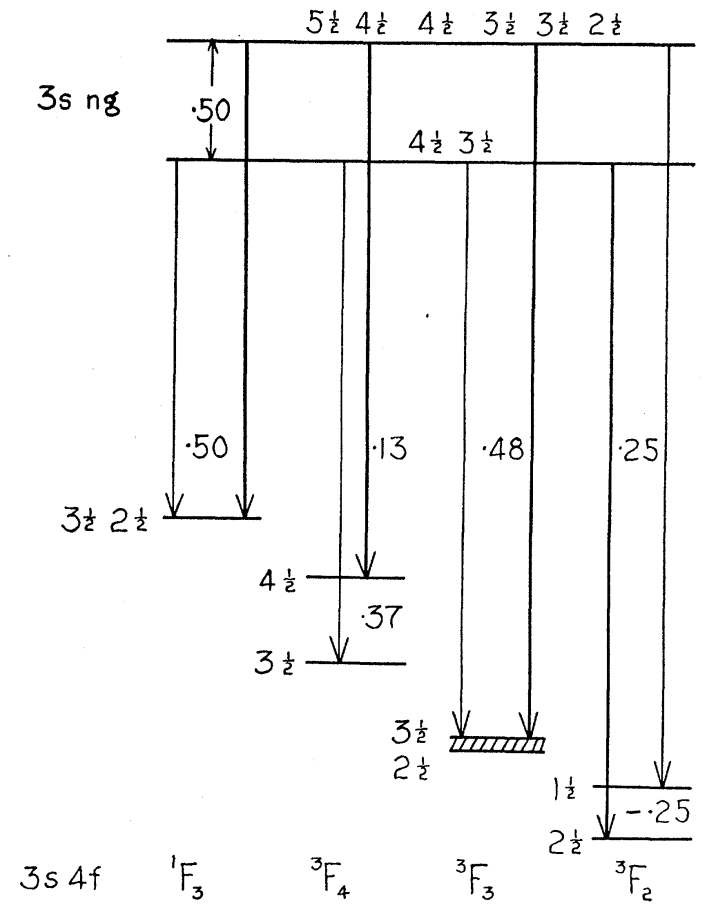

FIG. 4. Interpretation of hyerpfine structure of the transitions $3 s \mathrm{n} g-3 s 4 f$ in $\mathrm{Al}$ II.

and ${ }^{3} F_{2}$ they are also parallel and these levels will therefore combine principally with the upper of the $G$-levels. The same reasoning shows that the lower $G$-level gives transitions to the lowest levels of ${ }^{3} F_{4}$ and ${ }^{3} F_{2}$ as shown in Fig. 4 . For the other states nothing can be said with certainty from such simple considerations, but because of the fact that the ${ }^{3} F_{3}$ and ${ }^{1} F_{3}$ separations are expected to be so small, only two lines are observed anyway. For the relative intensity of the lines which are found, one would expect, from the intensity sum rules looking only at the lower levels ${ }^{3} F_{4}$ and ${ }^{3} F_{2}$, that those lines to the $F=4 \frac{1}{2}$ and $F=2 \frac{1}{2}$ would be stronger. For ${ }^{3} F_{3}$ and ${ }^{1} F_{3}$ there is no criterion of this sort from the lower levels but it might be expected that the stronger components will be those to the upper $G$ state as it has greater degeneracy. For the transition to ${ }^{3} F_{2}$ it will thus be the component of smallest frequency, for the others it will be that of largest frequency.

These considerations together with the expectation that the ${ }^{1} F_{3}$ should have no observable hyperfine structure leads to the interpretation of the observations given in Fig. 4. The $3 s \mathrm{ng}$ 
states are split up into two unresolvable groups about $0.50 \mathrm{~cm}^{-1}$ apart. The separations of the $3 \mathrm{~s}$ $4{ }^{3} F_{4},{ }^{3} F_{3},{ }^{3} F_{2}$ and ${ }^{1} F_{3}$ are about $0.37, \sim 0$, -0.25 , and $0 \mathrm{~cm}^{-1}$, respectively. The separation of the ${ }^{3} F_{4}$ state is still considerably larger than expected, but this may well be due to the approximate nature of our considerations in which we neglect entirely a possible further splitting of the $3 s \mathrm{n} g$ states.

In support of this interpretation it can be said that the hyperfine structure has only been resolved in the transitions from the $3 s 8 g, 3 s 9 g$ and $3 s \quad 10 g$ levels. The $3 s 7 g$ shows a further splitting up which causes the pattern to become too complicated and the lines are not resolved but denoted as "diffuse." The value obtained for $a$ is furthermore in good agreement with that from the preliminary data of Ritschl.

\section{APPENDIX}

Since this paper was written, an article by Professor F. Paschen ${ }^{11}$ has been received in

\footnotetext{
${ }^{11}$ Paschen, Sitz. Ber. Preuss. Akad. 1932.
}

which he treats qualitatively some of the questions considered here and which contains valuable new data on aluminum. He points out that the forbidden transition $6 s 6 d^{1} D_{2}-6 s 7 p^{3} P_{0}$, $\lambda 2967.5$, in $\mathrm{Hg} \mathrm{I}$ is due to hyperfine structure perturbations similar to a Paschen-Back effect and should occur only for the odd isotopes. Furthermore Professor Paschen discusses again the hyperfine structure of the $3 s 4 f$ levels in Al II and shows that his previous interpretation must be altered in order to take into account the hyperfine structure of the $3 s \mathrm{ng}$ series, which he finds to be larger than the ordinary multiplet structure and singlet-triplet distance.

The results of Professor Paschen on Al II do not quite agree with those given here, as he supposes the separation of the $6 s \mathrm{n} g$ levels to be $0.35 \mathrm{~cm}^{-1}$. For the forbidden transitions in mercury it might be mentioned that the lines $6 s 7 d^{1} D_{2}$ and $6 s 8 d^{1} D_{2}-6 s 7 p^{3} P_{0}, \lambda 2536.04$ and $\lambda 2379.46$ are difficult to explain since for these levels the perturbations are much smaller than for $6 s 6 d$. 